\title{
A Research on Link Mining Tasks
}

\author{
Zaved Akhtar, Ravindra Kumar, Umesh Chandra Jaiswal, Davesh Kumar Sharma
}

\begin{abstract}
Coping with the difficulty of mining luxuriously prepared, heterogeneous datasets wherein the devices are linked together are massive problems of records mining. Connections between gadgets may also show effective examples which can be useful for some form of statistics mining undertakings and are usually now not a smooth errand to trap with traditional authentic fashions. Some informational collections of intrigue nowadays are quality depicted as a connection accumulation of bury associated objects. These articles communicate to homogeneous systems in which there are single-object kind and connection kinds (eg. People associated with the aid of partner's connections or the arena wide net, accumulations associated internet site pages) or more extravagant heterogeneous systems wherein there might be numerous articles, conceivably distinct semantic data and connection types. In medicinal location portraying patients, infections, medications, and contacts, or in bibliographic regions, as an example, depicting productions, creators and scenes are the times of heterogeneous structures. Connection mining is an information mining structures that unequivocally bear in mind the ones association of connections even as constructing classificatory or premonition models of the related records. Normally connection mining assignments include article positioning, mixture arrangement, interface forecast, bunch recognition and sub-diagram revelation. It's far a provocative and fast developing location. In this examine paper we survey connection mining errands, a few primary developing subjects and talk approximately non-stop connection mining issues, open dispute and endorse mind that is probably open doors for preparations. The maximum stop of this take a look at is to provide future levels in link Mining and proposes mind to apply connection mining structures from connection mining to enlarge Semantic net.
\end{abstract}

Index Terms-Data Representation, Link Prediction, Graph Classification, Semantic Web.

\section{INTRODUCTION}

Link mining is a information mining strategies that explicitly don't forget a hyperlink or series of links for constructing predictive or descriptive fashions of the related statistics. Normally links mining obligations consist of, organisation detection, object rating, hyperlink predictions, collective kind, graph and sub-graph discovery. It additionally represents an essential and crucial set of strategies for constructing useful programs of data mining in a big type of real and important domains, in particular that concerning complex occasion detection from fantastically based data.

Tackling the problems of mining richly and extensively dependent heterogeneous datasets are most important rising mission for statistics mining. The ones kinds of datasets are awesome defined as networks, graphs or sub-graphs. In

Revised Manuscript Received on April 12, 2019.

Zaved Akhtar, Research Scholar, Dr. APJ AKTU, Lucknow. India (javed.gkp@rediffmail.com)

Ravindra Kumar, (Ex) Director , NIET, Gr. Noida. India (rkumar_viet@rediffmail.com)

Umesh Chandra Jaiswal, Professor(CSE Deptt.) \& Registrar, MMMUT, Gorakhpur. India (ucj_jaiswal@yahoo.com) Ghaziabad, UP. India (er_davesh@yahoo.co.in)
Davesh Kumar Sharma, Assistant Professor(CSE Deptt.), SRMIST,

these domains regularly encompass a diffusion of object kinds; and people gadgets can be related in a terrific ways. Because of this, we are capable to mention that the graph can also have specific node and component (or hyper-side) kinds. Interest must be taken all through those ability correlations because of hyperlinks are dealt with as it should be. Indeed, item linkage is statistics that want to be taken inside the shape of benefits. This kind of statistics may be used to decorate the revelation accuracy of the located fashions: attributes which is probably used in linked gadgets are regularly correlated and hyperlinks are much more likely to exist between items that have some common features. Similarly, we are able to say that the graph shape itself may be an essential element to include within the version. Structural homes along with connectivity and diploma may be crucial signs.

Among statistics instances, links or relationships are ubiquitous. Typically these hyperlinks show off styles that can mean homes of the records instances along with the importance, category, or rank of the item. In some instances, we cannot observed all links. Therefore, we may be inquisitive about

Predicting the perseverance of hyperlinks among instances. In distinctive domains, in which the hyperlinks are emerging over the years, our intention may be to are looking forward to whether a hyperlink will exist in the destiny, on the premise of previously discovered links. Thru taking hyperlinks into an account this results in a few different venture focused on discovering substructures, which includes groups, businesses, or not unusual sub graphs.

Link mining is a newly rise studies vicinity it's the intersection of link assessment [1], [2], hypertext and internet mining [3], relational analyzing, inductive common sense programming [4] and graph mining [5]. We use the term link mining is to put a unique hobby on the hyperlinks -shifting them as lots as first elegance citizens in the statistics evaluation exertion. In current years, there had been numerous workshop collection prepared and absolutely devoted to topics associated with hyperlink mining [1]. Exceptional workshop collection include the workshops on "Statistical Relational mastering, Multi- Relational statistics Mining" [6], [7], "hyperlink KDD" [8], [9], hyperlink analysis, Counter-terrorism and protection [10], Mining Graphs, wood and Sequences [11].

The purpose of this survey is to offer an outline on research in the applicable agencies which can be addressing modern link mining stressful situations. Link mining having a good sized variety of duties; therefore our examine will cowl the center annoying situations addressed by using a majority of ongoing research in this concern. Starting by

Published By: 


\section{A Research On Link Mining Tasks}

manner of describing a number of the middle demanding situations in statistics representation for hyperlink mining. Then we progress thru link mining obligations. Finally, we summaries with a discussion of areas that we accept as true with have no longer however acquired sufficient interest and advise mind and scopes that would be opportunities for answers.

\section{DISTURBING CONDITIONS IN FACTS INSTANCE}

In a conventional gadget reading algorithms feature choice and facts instance are large troubles; related data instance is even greater complicated. Bear in mind an instance of a social community describing a relationship among actors and their participation in sports. Such form of social networks are so usually known as association networks and are without problems represented by way of within the shape of 3 tables representing the actors, the sports and the participation relationships. Even it can be represented by way of the usage of several wonderful graphs. The bipartite graph is a maximum natural illustration approach, with a set of actor nodes, a set of occasion nodes, and set of edges that represent an actor's participation in an event. Each different approach for representations might also moreover allow high-quality insights and evaluation. As an instance, we can also moreover assemble a network, in which the nodes are actors and edges correspond to actors who've participated in an occasion together. This sort of illustration lets in us to perform a extra actor-centric evaluation. Alternatively, the ones family individuals we can also constitute as a graph, wherein the activities are nodes, and occasions are associated if an actor is in commonplace. Consequently the suitable preference of facts instance in link mining is absolutely a primary issue, and is often more complex than in the case if we've were given IID information times.

\section{HYPERLINK MINING DUTIES \& RESULTS}

Hyperlink Mining obligations are as comply with:

\section{A. Hyperlink Prediction}

Hyperlink Prediction isn't a clean undertaking, specifically whilst if it is approximately the future social networks are dynamic. Hyperlink prediction are used to count on future viable links within the community or anticipate missing links due to incomplete data (E.G., In meals-webs - this is related to sampling that Olivia mentioned in advance). New interplay between items indicating new links between them. Primarily based mostly on attributes of the gadgets and different observed links, link prediction is a trouble of predicting the lifestyles of a hyperlink among entities. A. Popescul et al.[24] added a dependent logistic regression version which could assume the lifestyles of links thru the rent the relational functions.

Examples embody including in social community predicting links amongst actors (E.G., facebook) including predicting friendships; predicting the participation of actors in activities. Distinctive examples which encompass emails, co-authorship and get in touch with calls; and predicting the semantic relationships like "consultant-of" based on their contents and available net page links [31].

\section{B. Sub-graph Discovery}

Sub-graph discovery or identification well-knownshows traits sub-graphs inner networks. A segment of information mining it truly is explored to link mining is the artwork on subgraph discovery. This paintings makes an attempt to seek typically or hobby grabbing incidence of sub-graphs in a tough and rapid of graphs. Discovery of those patterns is likewise used for graph class or is the only cause of the structures.

\section{Graph classification}

Graph elegance may be a supervised getting to know drawback or trouble interior which the purpose is to categorize an entire graph as a terrific or negative example of a idea. That is often one in every of the earliest obligations self addressed inside the context of applying gadget studying and facts mining / processing techniques to graph information. Graph class doesn't usually need collective logical wondering, as is needed for classifying items and edges, due to the truth the graphs are generally assumed to be independently generated.

3 fundamental methods to graph type are supported characteristic mining on graphs, inductive common feel programming (ILP), and defining graph kernels. Characteristic mining on graphs uses strategies related to the ones delineate within inside the preceding section on subgraph discovery, mining of functions / options on graphs are occasionally finished through locating all frequent or informative sub systems inside the graph instances. These sub systems are every now and then used for remodeling / transforming the graph information into statistics described as a unmarried desk and then conventional / historic classifiers are used for classifying the instances.

\section{Link-based totally item ranking}

Another link Mining mission is that of hyperlink-based absolutely object ranking. Number one reputation of linkbased item ranking is the link analysis community. The goal of link based totally object ranking is to make the most the link structure of a graph an excellent manner to prioritize the to be had set of gadgets inside the graph. HITS and internet page rating are most amazing techniques for hyperlink primarily based object score.

Within the viewt of net statistics retrieval, the PageRank [12] and HITS [13] algorithms are the very most remarkable approaches to hyperlink based absolutely object rating. In a web page ranking models, web browsing can be taken as random walk in which the surfer randomly selects and follows hyperlinks and infrequently switch to a brand new net internet web page to begin every other traversal of the based hyperlink. The rank provided to a web net web page for the duration of this context is that of partition of time that the random web surfer could spend on the web page if the random strategies have been iterated at infinity. This work may be determined via computing the regularstatedistribution of the random techniques or procedures.

Okay. Bharat and M. R. Henzinger [23], and S. Chakrabarti et al [29] proposed changes to HITS that make the maximum internet web web page information to weight 
pages and hyperlinks supported in step with relevancy of the pages. $\mathrm{Ng}$ analyzes the sturdiness or stability of PageRank and HITS to little perturbations within the hyperlink structure and proposed adjustments to HITS that yield more solid scores. T. H. Haveliwala [27] and Jeh and Widom [28] endorse subject matter-sensitive PageRank algorithms which has set up locally authoritative net pages expeditiously at query time.

In context to social community evaluation (SNA), link Bases object ranking is a key assessment assignment. The goal is to rank order in my opinion in a given social community in phrases of calculate of their significance, referred to as centrality.

\section{E. Hyperlink-based totally object classification}

In a conventional approach, gadgets are categorised at the concept of their attributes that describe them. Link-based totally totally category forecast the elegance of an object based on its attributes, and also on its hyperlinks and at the attributes of connected gadgets. Traditional device studying has attracted on the kind of records having identically established objects which might be typically to be assumed in IID. However, in plenty of real-world datasets, we lack this type of homogeneity of structure. Inside the hyperlink primarily based completely item class troubles, a information graph $\mathrm{G}=(0 ; \mathrm{L})$ is a composition of collected several set items zero and are related to each $\mathrm{G}=(0 ; \mathrm{L})$ it's combination of a difficult and speedy gadgets 0 and are related to every different through a hard and rapid of several links L. The responsibilities want to be labeling the contributors of 0 from the finite set of specific values. In plenty of instances, a feature of link based item Classifications makes it specific from traditional classifications and the labels of associated devices are to be correlated.

In modern-day years, hyperlink primarily based item elegance has received big observations. S. Chakrabarti, B. Dom, and P. Indyk [36] taken into consideration the hassle of classifying associated new devices within the related dataset. They have been the primary who targeted on their research paper that on exploiting beauty labels of associated gadgets aids elegance, wherein exploiting features of items which can be related to them can genuinely harm classification accuracy. Similar consequences had been primarily based with the useful resource of Oh on a group of encyclopedia articles: truely using the predicted class of neighboring files were beneficial even as incorporating phrases from neighboring documents had been now not. Lafferty introduce a new conditional random fields (CRF), which expand conventional most entropy models for link based object class inside the confined case where the data graphs are chains. B. Taskar, P. Abbeel, and D. Koller [30] make bigger Latterly's method to the case in which the information graphs are arbitrary graphs. Another researcher Neville proposed a clean and novel hyperlink primarily based definitely object kind set of rules for classifying organization datasets with richly primarily based schemas that produces graphs with heterogeneous items wherein every body with its personal wonderful set of competencies. $\mathrm{Lu}$ and Getoor [2], [25] extends easy device mastering classifiers to carry out link based totally object
Classifications thru introducing new features to measures the distributions of sophistication labels in the Markov blanket of the item which have to be classified.

\section{A Link-Based Cluster Analysis}

The primary reason of link-based totally cluster evaluation is to discover going on subclasses. That is finished with the resource of clustering the whole records into organizations, where gadgets in a set are just like each other and are very numerous from the gadgets which might be in unique organizations. Unlike category, clustering is unsupervised and may be implemented to discover hidden patterns from statistics. This makes it a honestly ideal method for packages like semantic records exploration, statistics retrieval, computational biology, net log based assessment, crook evaluation and lots of others.

There has been intensive research art work on cluster in regions like pattern reputation, records and device gaining knowledge of. Hierarchical agglomerative clustering (HAC) and ok-way are 2 of the maximum common cluster algorithms. Probabilistic version-based totally cluster is gaining growing first rate and recognition [14; 15]. All of those algorithms assume that each object is delineated thru a difficult and fast period characteristic-price vector.

\section{B. Organization Detection}

Organization detection is a few other cluster challenge. It predicts once cluster of gadgets belong to the same agency or cluster, supported their set of attributes moreover as their hyperlink shape. As an example organization detection is to cluster the nodes of a graph into company or groups which percentage not unusual developments. In contemporary years, there was a primary undertaking to expand scalable methods that would take gain of an increasing number of complex graphs to resource the records discovery method.

Don't forget first the case in which a graph includes numerous gadgets and hyperlinks of a single kind, with out attributes. Numerous techniques for identifying businesses on this state of affairs may be categorised as both divisive or agglomerative clustering techniques.

The intention to create a block modeling of social network evaluation (SNA) includes segmenting social networks into the devices of people which can be called positions that display off homogenious devices of hyperlinks to others within the network. A similarity measures are described among agglomerative clustering and they used to identify the positions. Spectral Graph segmentation techniques cope with the organization detection problem by means of manner of identifying an approximately minimal set of hyperlinks to take away from the graph to obtain a given extensive type of groups [16]. In a associated vein, D. Gibson, J. Kleinberg, and P. Raghavan [17] have verified that the ascends eigenvectors of the HITS authority matrix offer a herbal decomposition of net network shape. One-ofa-kind cutting-edge strategies that are used for organization detection uses a degree of side betweenness, derived from Freeman's belief of betweenness centrality, to turn out to be privy to hyperlinks connecting corporations [18]. Excessive 


\section{A Research On Link Mining Tasks}

aspect links among them are additionally eliminated for the motive of partitioning the graph.

\section{Entity selection}

Entity resolution is each other object-centric mission of hyperlink mining, which involves to identifying the set of objects in a internet site. The primary goal of entity resolution classify that which references within the data consult with the identical real-international entity.

For the reason of decision, hyperlink emerge as first explored in databases. R. Ananthakrishna, S. Chaudhui and V. Ganti [37] proposed a way using hyperlinks for reduplication in records warehouse packages wherein there exist dimensional hierarchy over the hyperlink members of the circle of relatives. D. V. Kalashnikov, S. Mehrotra, and Z. Chen [19] higher characteristic-primarily based totally similarity amongst an ambiguous reference and the severa entity alternatives for it with linkage evaluation a number of the entities, such as affiliation and co-authorship. In database [20; 21] collective entity selection procedures have moreover been proposed wherein one resolution choice influences every other if they may be related. Bhattacharya and Getoor [20];[ 22] advise wonderful measures for linkage similarity in graphs and display how the ones can be combined with characteristic similarity for collective entity selection in collaboration graphs. X. Dong, A. Halevy, and J. Madhavan [21] collectively treatment entities of more than one kinds with the aid of the usage of propagating proof over links in a very dependency graph.

II. OPEN troubles AND research areas

In middle pattern discovery and pattern detection, the winning actual packages usually have several requirements. Requirement arises from the characteristics of the environment; e.G., the need to mix statistics from more than one assets, the need to compute few derived attributes to be utilized by way of the pattern discovery and pattern detection algorithms, , the want to file and audit tool sports, the want to manual more than one analysts, the want to type facts into amazing threads of hobby, the want to visualize patterns (in every senses of the term), the want to support detection of patterns which could occur over long time periods, the want to each discover and find out styles which can be continually changing, the need to comfortable the identification of entities till a suitable stage of perception in their interestingness is supported and, possibly, proper approvals are received, the need to allow for more than one competitor hypotheses, the requirement to permit for commonplace standardization among fake positives and fake negatives, the requirement to allow for refutation of antecedently declared evidence, the requirement to assist every complete assessment of all facts based totally mostly on supported authorised styles and ad-hoc assessment supported unique external signs and symptoms, the want to useful resource structural / organizational workflow manner, the need to resource what-if analyses through humans, the need to function constantly and can be autonomously on incrementally arriving big records streams, and probable others. Other than these, there are numerous other analytical research troubles exist. We suggested some mind for future artwork which is probably as follow:
A. Question-primarily based completely class using hyperlink

The usage of links, query-based elegance is a exquisite research directional region. While customers are inquisitive about unique vicinity or set of contents, it's far tin pot to provide big amount of data to them. In kind approach for ponder the dataset in its entire jointly associated instance of item which can carry out prediction or kind for all of those associated items. As soon as a person is curious approximately classifying solely a small subset of those gadgets, it's worth to classify alternate devices supplying they're beneficial in well classifying the items of hobby via the link structure.

\section{B. Characteristic production}

In multi-relational placing, function incorporation and protection is a extraordinary mission. The attributes of an object gives a number one description of the item. Conventional magnificence algorithms are based on the ones styles of object features. While we use hyperlink-based approach, it can additionally make revel in to apply attributes of related gadgets. Moreover if the links themselves having set of attributes then those also can be used. This is the idea at the back of propositionalization [32]. However, as others have referred to, absolutely knocking down the relational community around an object can be elaborate. Numerous have mentioned that during hypertext domain names, without a doubt including phrases from neighboring pages degrades class overall performance [33]. We've were given determined this works well for learning probabilistic relational fashions [34], however this technique might not continuously be appropriate so there may be a want to regulate and improve probabilistic relational fashions for feature choice.

\section{Net searching for \& Retrieval}

Any other objective of link mining techniques used for web are trying to find and retrieval the text in addition to information efficiently.

\section{Hyperlink Mining strategies to discovering patterns and assemble beneficial prediction device}

Now an afternoon's quantity of information grows and the extensive style of assets expands very swiftly, strategies from link mining can come to the useful aid of us for discover styles and construct useful prediction system. We advised to the usage of hyperlink mining strategies to increase a sample discovery and prediction device to infer establishments among gadgets it is useful to discover web character surfing styles, patron agencies and so on.

\section{E. Using hyperlink Mining strategies to assemble Semantic internet}

Now an afternoon, internet search results are high recall, low precision and consequences are tremendously touchy to vocabulary. The Semantic internet is an internet of facts wherein statistics are properly-defined manner, precise or approx similarly to offer higher allowing laptop systems and customers to art work in co-operation. 
The Semantic internet is set topics, describes the relationships amongst these objects (like $\mathrm{A}$ is part of $\mathrm{B}$ and $\mathrm{Y}$ is a member of $\mathrm{Z}$ ) and the houses of factors (like age, weight, length and charge).There are commonplace codecs for mixture and integration of facts drawn from diverse resources, wherein at the particular internet particularly targeting the interchange of files. It's also about the used languages for recording how the facts relates to real international gadgets further to permits someone, or a machine, to start off in a single database, after which flow thru an unending set of databases which are linked now not by using wires but by way of being about the identical factor. The Semantic net is not approximately hyperlinks among pages, it describes the relationships and houses between things.

We proposed to coming across interesting sub-graph based totally on semantic records associated with the rims and combining records extraction with the help of hyperlink Mining strategies to collect the Semantic net.

\section{CONCLUSION}

To molding and tending deeply connection mining problems, there are fundamental and vital development has been made as of late, but a wonderful deal art work remains to be performed in refining and consolidating precise methodologies and preparations. The maximum putting cease of this paper end of this that there are some connection mining strategies that competencies admirably for man or woman or specific connection mining errands but no longer yet a radical form or tool which can also bolster a mixture of connection mining assignments that are required in numerous applications. To development of effective and accommodating connection mining applications is stays stay a take a look at. Connection mining errands and difficulties offer fascinating bits of know-how and catalyze new studies bearings. To concocting or constructing up a skilled design to assist each unmarried important potential of associated blanketed application and giving a reaction to the use connection digging structures for Semantic net is furthermore a key to progress.

\section{REFERENCES}

1. D. Jensen and H. Goldberg, "computer based completely intelligence and link analysis"., AAAI Fall Symposium, AAAI Press, 1998.

2. L. Getoor, "Connection Mining a new facts Mining mission", SIGKDD Explorations, 5 (1), pp. Eighty four-89, 2003.

3. Peng Wang, Wen XUBao, Yurong WU and XIO Yu Zhou, " link Prediction in Social Networks: The contemporary day", Cornel university library, facts Sciences, China, Vol fifty eight, pp. 011101:1-011101:38, January 2015.

4. S. Dzeroski and N. Lavrac, " Relational records Mining", Kluwer, Berlin, 2001.

5. D. J. Cook and L. B. Holder, "Chart based facts mining". IEEE smart structures, 5(2), pp. 32-41, 2000.

6. T. Dietterich, L. Getoor, and k. Murphy, " ICML paintingssave on Statistical Relational learning and its Connections to different Fields", 2004.

7. S. Dzeroski and H. Blockeel, "KDD Workshop on MultiRelational information Mining", 2004.

8. Anshu Zhang, Wenzhung Shi, Geoffery, I. Webb, "Mining giant affiliation rules From unsure data", information Mining and knowledge Discovery, Vol 30, ISSN: 1348-5810, pp. 928963, July 2016.

9. J. Adibi, H. Chalupsky, M. Grobelnik, N. Milic-Frayling, and D. Mladenic, ".KDD Workshop on link analysis and business enterprise Detection", 2004.

10. D. Skillicorn and okay. Carley, "SIAM Workshop on link assessment, Counterterrorism and safety", 2005.

11. P. Bhattacharya, A. Garg and F. S. Wig:, "exam of man or woman key-word Similarities in online Social Networks", Social network assessment and Mining,, pp. 143-158, 2011.

12. L. Web page, S. Brin, R. Motwani, and T. Winograd, "The PageRank reference positioning: Bringing request to the we", Technical document, Stanford college, 1998.

13. J. Kleinberg, "Definitive assets in a hyperlinked situation", magazine of the ACM, forty six(5), pp. 604-632, 1999.

14. B. Taskar, E. Segal, and D. Koller, " Probabilistic affiliation and grouping In social records" IJCAI-01, 2001.

15. J. Kubica, A. Moore, J. Schneider, and Y. Yang, " Stochastic connection and collecting identity", AAAI-02, 2002.

16. M. E. J. Newman, " Detecting people institution structure in systems", ecu physical magazine B, 38, pp. 321-330, 2004.

17. D. Gibson, J. Kleinberg, and P. Raghavan, "Deriving internet networks from connection topology", In ACM conference on Hypertext and Hypermedia, pp. 225-234, 1998.

18. J. R. Tyler, D. M. Wilkinson, and B. A. Huberman, "electronic mail as Spectroscopy: automated Discovery of network shape internal agencies", Kluwer, B.V., Deventer, The Netherlands, The Netherlands, 2003.

19. D. V. Kalashnikov, S. Mehrotra, and Z. Chen, "Misusing connections for place loose statistics cleanin", SIAM international convention on facts Mining, April 21-23, 2005.

20. I. Bhattacharya and L. Getoor, "Iterative record linkage for cleansing and blend", In SIGMOD 2004 Workshop on studies troubles on statistics Mining and expertise Discovery, June 2004.

21. X. Dong, A. Halevy, and J. Madhavan, "Reference compromise in complex records space", In ACM SIGMOD global conference on control of records, pp. 80 5-ninety six, 2005.

22. I. Bhattacharya and L. Getoor, " Entity goals in charts", Technical report 4758, computer technological understanding department, college of Maryland, 2005.

23. k. Bharat and M. R. Henzing, "improved calculations for issue refining in a hyperlinked location", In ACM SIGIR global convention on studies and improvement in statistics Retrieval, pp.. 104-111, 1998.

24. A. Popescul and L. H. Ungar, "Statistical social getting to know for connection expectation", In IJCAI Workshop on getting to know Statistical models from Relational records, 2003.

25. Lu, Q, Getoor, L., "Connection based category". ICML'03, Washington DC, 2003.

26. Sen, P., Getoor, L., "Connection primarily based elegance". College of Maryland CS-TR, pp. 48-fifty eight, 2007.

27. T. H. Haveliwala, "theme sensitive PageRank", In worldwide convention on the sector extensive internet (WWW), pp., 517 526, 2002.

28. G. Jeh and J. Widom, " Scaling customized internet search", In worldwide conference on the arena big net (WWW), pp. 271-279, 2003

29. S. Chakrabarti, B. Dom, D. Gibson, J. Kleinberg, P. Raghavan, and S. Rajagopalan, "Programmed asset list aggregation via investigating link form and associated content material", In worldwide international big net conference (WWW), 1998.

30. B. Taskar, P. Abbeel, and D. Koller, "Discriminative probabilistic models for social data", In Proc. Of UAI, pp. 485-492, Edmonton, Canada, 2002. 
31. B.Taskar, M.- F.Wong, P.Abbeel and D.Koller, "Connection forecast in social statistics", In Neural facts Processing structures conference, Vancouver, Canada, December 2003.

32. S. Kramer, N. Lavrac, and P. Flach, "Propositionalization techniques to address social information minin", In S. Dzeroski and N. Lavrac, editors, Relational data Mining, pp. 262 - 291. Kluwer, 2001

33. H.- J. Goodness, S. H. Myaeng, and M.- H. Lee, " an inexpensive hypertext association approach the usage of joins and steadily handy elegance records", In intending. Of SIGIR00, 2000.

34. L. Getoor, N. Friedman, D. Koller, and A. Pfeffer, "gaining knowledge of probabilistic social models", In S. Dzeroski and N. Lavrac, editors, Relational information Mining, pp 307 335. Kluwer, 2001

35. D. Jensen, " Statistical difficulties to inductive surmising in connected facts", In 7th international Workshop on artificial Intelligence and facts, 1999.

36. S. Chakrabarti, B. Dom, and P. Indyk, "Upgraded hypertext order utilising hyperlink", In SIGMOD international conference on management of information, pp. 307-318, 1998.

37. R. Ananthakrishna, S. Chaudhui and V. Ganti, "removing fluffy copies in data stockrooms", In worldwide conference on Very big Databases (VLDB), Hong Kong, China, 2002. 